\title{
Existence and stability of almost periodic solutions for impulsive differential equations
}

\author{
Junwei Liu ${ }^{*}$ and Chuanyi Zhang
}

* Correspondence: junweiliuhit@gmail.com Department of Mathematics, Harbin Institute of Technology, Harbin 150001, PR China

\begin{abstract}
In this article, by using Schauder's fixed point theorem, we study the existence of almost periodic solutions for abstract impulsive differential equations. In addition, sufficient conditions for their asymptotic stability are obtained by means of generalized Gronwall-Bellman inequality.
\end{abstract}

Keywords: abstract impulsive differential equations, Schauder's fixed point theorem, asymptotic stability, generalized Gronwall-Bellman inequality

\section{Introduction}

When a dynamical system processes aftereffect and state changing by jumps, this system is called an impulsive differential system. Because of the needs of modern technology, such as simulation in physics, biology, populations dynamics, control theory, industrial robotics, etc., the study of impulsive differential equations attracts more and more researchers' interest, see [1-8]. There are many articles [9-16] about existence of solutions, periodic solutions and stability for impulsive differential equations. But, only a few articles $[17,18]$ have studied the existence of almost periodic solutions to abstract impulsive differential equations in Banach space.

By means of fractional powers of operators and Contraction mapping principle, Stamov and Alzabut in [17] studied the existence and uniqueness of exponentially stable almost periodic solutions for the abstract impulsive differential equation as follows:

$$
\dot{x}(t)+A x=F(t, x)+\sum_{k=-\infty}^{\infty} G_{k}(x) \delta\left(t-\tau_{k}\right),
$$

where $G_{k}(\cdot)$ are continuous impulsive operators.

In [18], by using Contraction mapping principle, Henriquez et al. studied the existence of almost periodic solutions to the following impulsive differential equation:

$$
\left\{\begin{array}{l}
u^{\prime}(t)=A(t) u(t)+F(u(t))+f(t), t \in R, t \neq t_{i}, i \in Z, \\
\Delta u\left(t_{i}\right)=I_{i}\left(u\left(t_{i}\right)\right) .
\end{array}\right.
$$

To the best of authors' knowledge, most of the previous research on existence of almost periodic solutions for impulsive differential equations was based on Contraction mapping principle. Lipschitz condition is necessary. Motivated by the studies in $[8,12,13,19-21]$, in this article we use Schauder's fixed point theorem and generalized 
Gronwall-Bellman inequality to investigate the existence and asymptotic stability of almost periodic solutions to the following impulsive differential equation:

$$
\left\{\begin{array}{l}
u^{\prime}(t)=A(t) u(t)+f(t, u(t)), t \in R, t \neq t_{i}, i \in Z, \\
\Delta u\left(t_{i}\right)=I_{i}\left(u\left(t_{i}\right)\right)
\end{array}\right.
$$

where $A(t): X \rightarrow X$ are closed linear operators on a Banach space $(X,\|\cdot\|), f, I_{i}$, and $t_{i}$ satisfy suitable conditions that will be established later and the symbol $\Delta \xi(t)$ represents the jump of the function $\xi$ at $t$, which is defined by $\Delta \xi(t)=\xi\left(t^{+}\right)-\xi\left(t^{-}\right)$.

\section{Preliminaries}

In this section, we give some notations, definitions, and preliminary facts about impulsive differential equations. Throughout this article, we denote by $(X,\|\cdot\|)$ a Banach space and by $R$ the set of real numbers. The symbol $L(X)$ stands for the Banach space formed by all bounded linear operators form $X$ into $X$ endowed with the uniform operator topology. For a linear operator $A, \rho(A)$ is its resolvent set and for $\lambda \in \rho(A), R$ $(\lambda, A)=(\lambda I-A)^{-1}$ denotes its resolvent operator. Let $\mathbb{T}$ be the set consisting of all real sequences $\left\{t_{i}\right\}_{i \in Z}$ such that $\alpha=\inf _{i \in Z}\left(t_{i+1}-t_{i}\right)>0$ and $\lim _{i \rightarrow-\infty} t_{i}=-\infty, \lim _{i \rightarrow-\infty} t_{i}=\infty$.

For $\left\{t_{i}\right\}_{i \in Z} \in \mathbb{T}$, let $P C(R, X)$ be the space formed by all piecewise continuous functions $\varphi: R \rightarrow X$ such that $\varphi(\cdot)$ is continuous at $t$ for any $t \notin\left\{t_{i}\right\}_{i \in Z}$ and $\phi\left(t_{i}\right)=\phi\left(t_{i}^{-}\right)$ for all $i \in Z$; let $P C(R \times X, X)$ be the space formed by all piecewise continuous functions $\varphi: R \times X \rightarrow X$ such that for any $x \in X, \varphi(\cdot, x)$ is continuous at $t$ for any $t \notin\left\{t_{i}\right\}$ $i \in Z$ and $\phi\left(t_{i}, x\right)=\phi\left(t_{i}^{-}, x\right)$ for all $i \in Z$ and for any $t \in R, \varphi(t, \cdot)$ is continuous at $x \in X$.

For a function $f \in P C(R, X)$ (respectively $P C(R \times X, X))$ and $\tau \in R$, the translation of $f$ by $\tau$ is the function $R_{\tau} f(t)=f(t+\tau)$ (respectively, $R_{\tau} f(t, x)=f(t+\tau, x)$ ).

A number $\tau \in R$ is called an $\varepsilon$-translation number of the function $f \in P C(R, X)(P C($ $R \times X, X))$ if

$$
\left\|R_{\tau} f(t)-f(t)\right\|<\varepsilon,\left(\left\|R_{\tau} f(f, x)-f(t, x)\right\|<\varepsilon, x \in K, \text { for every compact } K \subseteq X\right)
$$

for all $t \in R$ which satisfies $\left|t-t_{i}\right|>\varepsilon$. Denote $\mathbb{T}(f, \varepsilon)$ by the set of all $\varepsilon$-translation numbers of $f$.

Definition 2.1 (D1) A function $\varphi \in P C(R, X)(P C(R \times X, X))$ is said to be piecewise almost periodic if the following conditions are fulfilled:

(1) $\left\{t_{i}^{j}=t_{i+j}-t_{i}\right\}, j \in Z$, are equipotentially almost periodic.

(2) For any $\varepsilon>0$ (every compact subset $K$ of $X$ ), there exists a positive number $\delta=$ $\delta(\varepsilon)(\delta=\delta(\varepsilon, K))$ such that if the points $t^{\prime}$ and $t^{\prime \prime}$ belong to a same continuity and $\mid t$

' - $t^{\prime \prime} \mid<\delta$, then $\left\|\varphi\left(t^{\prime}\right)-\varphi\left(t^{\prime \prime}\right)\right\|<\varepsilon\left(\left\|\varphi\left(t^{\prime}, x\right)-\varphi\left(t^{\prime \prime}, x\right)\right\|<\varepsilon\right.$, for any $\left.x \in K\right)$.

(3) For every $\varepsilon>0$ (every compact subset $K$ of $X), \mathbb{T}(\phi, \varepsilon)(\mathbb{T}(\phi, \varepsilon, K)$ ) is a relatively dense set in $R$.

We denote by $A P_{T}(R, X)\left(A P_{T}(R \times X, X)\right)$ the space of all piecewise almost periodic functions. Obviously, the space $A P_{T}(R, X)\left(A P_{T}(R \times X, X)\right)$ endowed with the norm of the uniform convergence $\|\cdot\|$ is a Banach space.

(D2) A set $B \subseteq A P_{T}(R, X)$ is called a uniformly piecewise almost periodic family if it is uniformly bounded and if given $\varepsilon>0$, then $\mathbb{T}(B, \varepsilon)=\cap_{f \in B} \mathbb{T}(f, \varepsilon)$ is relatively dense in $R$. 
Obviously, if $f \in A P_{T}(R \times X, X)$ and for each compact set $K \subseteq X, f(t, x): R \times X \rightarrow X$ is uniformly continuous in $x \in K$ uniformly in $t \in R$, then $\{f(\cdot, x): x \in K\}$ is a uniformly piecewise almost periodic family

Lemma 2.2 [18] Let $\varphi \in A P_{T}(R, X)$, then the range of $\varphi, R(\varphi)$, is a relatively compact subset of $X$.

Lemma 2.3 If a set $B \subseteq A P_{T}(R, X)$ is relatively compact, then $\mathrm{U}_{f \in B} R(f)$ is a relatively compact set in $X$.

Proof Since $B$ is a relatively compact set, for any $\varepsilon>0$, there exists a finite number of functions $f_{1}, f_{2}, \ldots, f_{N} \in B$ such that for any $f \in B$, there exists a number $i \in\{1,2, \ldots$, $N\}$, then

$$
\left\|f-f_{i}\right\| \leq \frac{\varepsilon}{2} .
$$

By Lemma $2.2, R\left(f_{1}\right), R\left(f_{2}\right), \ldots, R\left(f_{N}\right)$ are relatively compact sets in $X$, so is $U_{1 \leq i \leq N} R\left(f_{i}\right)$, that is, $\mathrm{U}_{1 \leq i \leq N} R\left(f_{i}\right)$ can be embedded in a finite number of balls of radius $\frac{\varepsilon}{2}$. Let us denote by $x_{1}, x_{2}, \ldots, x_{n}$ the centers of the balls of radius $\frac{\varepsilon}{2}$ which cover the set $\mathrm{U}_{1 \leq i \leq N} R\left(f_{i}\right)$. For any $t \in R, f \in B$, let $x_{i}$ be the center of the ball of radius $\frac{\varepsilon}{2}$ which contains $f_{i}(t)$, we have

$$
\left\|f(t)-x_{i}\right\| \leq\left\|f(t)-f_{i}(t)\right\|+\left\|f_{i}(t)-x_{i}\right\| \leq \frac{\varepsilon}{2}+\frac{\varepsilon}{2}=\varepsilon .
$$

This show that $\mathrm{U}_{f \in B} R(f)$ is covered for any $\varepsilon>0$ by a finite number of balls of radius $\varepsilon$. This proof is complete.

For the operators $\{A(t): t \in R\}$, we make the following assumption $(\mathrm{H})$ :

(H1) $\{A(t): t \in R\}$ are linear operators on a Banach space $X$ and there are constants $K \geq 0, r \in R$ and $\phi \in\left(\frac{\pi}{2}, \pi\right)$ such that

$$
\|R(\lambda, A(t))\| \leq \frac{K}{1+|\lambda-r|}
$$

for $\lambda \in \rho(A(t)) \cap \Sigma(\varphi, r)$ and $t \in R$, where $\Sigma(\varphi, r)=\{r\} \cup\{\lambda:|\arg (\lambda-r)| \leq \varphi\}$.

(H2) There are constants $L \geq 0$ and $\mu, v \in(0,1]$ with $\mu+v>1$ such that

$$
|\lambda|^{v} \| A_{r}(t) R\left(\lambda, A_{r}(t)\right)\left(A_{r}(t)^{-1}-\left(A_{r}(s)^{-1}\right) \| \leq L|t-s|^{\mu}\right.
$$

for $A_{r}(t)=A(t)-r, t, s \in R$ and $|\arg \lambda|<\varphi$.

(H3) $R(\lambda, A(\cdot)) \in A P(L(X))$.

(H4) The evolution family $\{T(t, s): t, s \in R, t \geq s\}$ generated by $\{A(t): t \in R\}$ is exponentially stable, i.e., there exists numbers $M>0, w>0$, such that $\|T(t, s)\| \leq$ $M e^{-w(t-s)}, t \geq s, t, s \in R$.

(H5) For fixed $t, s \in R, t \geq s$, the operator $T(t, s): X \rightarrow X$ is compact, that is, $T(t, s)$ is continuous and maps a bounded set into a relatively compact set.

(H6) For each $x \in X, T(t+h, t) x \rightarrow x$ as $\mathrm{h} \rightarrow 0^{+}$uniformly for $t \in R$, i.e., $\{T(t, s): t$ $\geq s\}$ is uniformly continuous. 
Operators $\{A(t): t \in R\}$ fulfilling (H1) and (H2) are called sectorial, see [22-25].

Lemma 2.4 [25] Assume that the conditions (H1)-(H4) hold. Then for each $\varepsilon>0$ and $\delta>0$, there is a relatively dense set $\Omega_{\varepsilon, \delta}$ such that

$$
\|T(t+\tau, s+\tau)-T(t, s)\| \leq \varepsilon e^{-\frac{w}{2}(t-s)}, t-s>\delta, t, s \in R, \tau \in \Omega_{\in, \delta} .
$$

This property can be abbreviated by writing $T \in A P(L(X))$.

Lemma 2.5 [18] Assume that $f \in A P_{T}(R, X), T \in A P(L(X))$, the sequence $\left\{x_{i}: i \in Z\right\}$ is almost periodic, and $\left\{t_{i}^{j}\right\}, j \in Z$, are equipotentially almost periodic. Then for each $\varepsilon$ $>0$ there exist relatively dense sets $\Omega_{\varepsilon, f, x_{i}, T}$ of $R$ and $Q_{\varepsilon, f, x_{i}, T}$ of $Z$ satisfying:

(i) ||$f(t+\tau)-f(t)||<\varepsilon$ for all $t \in R,\left|t-t_{i}\right|>\varepsilon, \tau \in \Omega_{\varepsilon, f, x_{i}, T}$ and $i \in Z$.

(ii) $\|T(t+\tau, s+\tau)-T(t, s)\|<\varepsilon e^{-\frac{w}{2}(t-s)}$ for all $t, s \in R,|t-s|>0,\left|t-t_{i}\right|>\varepsilon,\left|s-t_{i}\right|$ $>\varepsilon, \tau \in \Omega_{\varepsilon, f, x_{i}, T}$ and $i \in Z ; w>0$ is the same as in (H4).

(iii) $\left\|x_{i+q}-x_{i}\right\|<\varepsilon$ for all $q \in Q_{\varepsilon, f, x_{i}, T}$ and $i \in Z$.

(iv) $\left|t_{i}^{q}-\tau\right|<\varepsilon$ for all $q \in Q_{\varepsilon, f, x_{i}, T}, \tau \in \Omega_{\varepsilon, f, x_{i}, T}$ and $i \in Z$.

Definition 2.6 A bounded function $u(t): R \rightarrow X$ is called a mild solution of (1.3) if for any $t \in R, \sigma<t_{i}<t<t_{i+1}$,

$$
u(t)=T(t, \sigma) u(\sigma)+\int_{\sigma}^{t} T(t, s) f(s, u(s)) d s+\sum_{\sigma<t_{i}<t} T\left(t, t_{i}\right) I_{i}\left(u\left(t_{i}\right)\right) .
$$

Under the assumption $(\mathrm{H}),\|T(t, \sigma)\| \leq M e^{-w(t-\sigma)}$ for all $t>\sigma$. Let $\sigma \rightarrow-\infty$, then $\| T$ $(t, \sigma) \| \rightarrow 0$ and so, the above formula can be replaced by

$$
u(t)=\int_{-\infty}^{t} T(t, s) f(s, u(s)) d s+\sum_{t_{i}<t} T\left(t, t_{i}\right) I_{i}\left(u\left(t_{i}\right)\right) .
$$

In fact, for $t>\sigma$,

$$
\begin{aligned}
& \int_{\sigma}^{t} T(t, s) f(s, u(s)) d s+\sum_{\sigma<t_{i}<t} T\left(t, t_{i}\right) I_{i}\left(u\left(t_{i}\right)\right) \\
& =\int_{-\infty}^{t} T(t, s) f(s, u(s)) d s-\int_{-\infty}^{\sigma} T(t, s) f(s, u(s)) d s \\
& \quad+\sum_{-\infty<t_{i}<t} T\left(t, t_{i}\right) I_{i}\left(u\left(t_{i}\right)\right)-\sum_{-\infty<t_{i}<\sigma} T\left(t, t_{i}\right) I_{i}\left(u\left(t_{i}\right)\right) \\
& =\int_{-\infty}^{t} T(t, s) f(s, u(s)) d s+\sum_{-\infty<t_{i}<t} T\left(t, t_{i}\right) I_{i}\left(u\left(t_{i}\right)\right) \\
& \quad-T(t, \sigma)\left[\int_{-\infty}^{\sigma} T(\sigma, s) f(s, u(s)) d s-\sum_{-\infty<t_{i}<\sigma} T\left(\sigma, t_{i}\right) I_{i}\left(u\left(t_{i}\right)\right)\right] \\
& =u(t)-T(t, \sigma) u(\sigma),
\end{aligned}
$$


so that,

$$
u(t)=T(t, \sigma) u(\sigma)+\int_{\sigma}^{t} T(t, s) f(s, u(s)) d s+\sum_{\sigma<t_{i}<t} T\left(t, t_{i}\right) I_{i}\left(u\left(t_{i}\right)\right) .
$$

The following lemma is a criterion of relative compactness for subsets in $A P_{T}(R, X)$, its proof is similar to the case in $A P(R, X)$ (e.g., see [26]).

Lemma 2.7 Let $\mathfrak{W} \subset A P_{T}(R, X)$. Then $\mathfrak{W}$ is relatively compact in $A P_{T}(R, X)$ if and only if the following properties hold true:

(i) $\mathfrak{W}$ is equicontinuous, which means that for any $\varepsilon>0$, there exists $\delta(\varepsilon)>0$ such that for any $f \in \mathfrak{W}$ one has $\|f(t)-f(s)\|<\varepsilon$ if $|t-s|<\delta(\varepsilon)$ and $t$ and $s$ in a same continuity.

(ii) $\mathfrak{W}$ is equi-almost-periodic, which means that for each $\varepsilon>0$ there exists $l(\varepsilon)>0$ such that any interval $(a, a+l) \subset R$ contains a number $\tau$ that is an $\varepsilon$ - almost period for all $f \in \mathfrak{W}$.

(iii) For fixed $t \in R$, the set $\{f(t): f \in \mathfrak{W}\}$ is relatively compact in the space $X$.

Definition 2.8 [27] The solution $x(t, \sigma, \varphi)$ of (1.3) is said to be stable, if for any $\sigma \in$ $R, \varepsilon>0$, there is a number $\delta=\delta(\sigma, \varepsilon)>0$, such that for any other solution $y(t, \sigma, \psi)$ of (1.3) satisfying $\|\phi-\psi\|<\delta$ then $\|x(t, \sigma, \phi)-y(t, \sigma, \psi)\|<\varepsilon$.

$x(t, \sigma, \phi)$ is said to be asymptotically stable if it is stable and if there is a constant $b>$ 0 such that $\|\phi-\psi\|<b$, then $\lim _{t \rightarrow \infty}\|x(t, \sigma, \phi)-y(t, \sigma, \psi)\|=0$.

Lemma 2.9 ([1], generalized Gronwall-Bellman inequality) Let a nonnegative function $u(t) \in P C(R, X)$ satisfy for $t \geq t_{0}$ the inequality

$$
u(t) \leq C+\int_{t_{0}}^{t} v(\tau) u(\tau) d \tau+\sum_{t_{0}<\tau_{i}<t} \beta_{i} u\left(\tau_{i}\right)
$$

where $C \geq 0, \beta_{i} \geq 0, v(\tau)>0$, and $\tau_{i}^{\prime} s$ are discontinuity points of first type of the function $u(t)$. Then the following estimate holds for the function $u(t)$,

$$
u(t) \leq C \prod_{t_{0}<\tau_{i}<t}\left(1+\beta_{i}\right) e^{\int_{t_{0}}^{t} v(\tau) d \tau} .
$$

\section{Main results}

In order to get the almost periodic solutions of (1.3), in addition to the previous assumptions, we require the following additional assumptions:

(A1) $\forall L>0, C_{L}=\sup _{t \in R},\|x\| \leq L \mid\|f(t, x)\|<\infty, C=\sup _{i \in Z}\left\|I_{i}\right\|<\infty$. Moreover, there exists a number $L_{0}>0$, such that $M\left(\frac{C_{L_{0}}}{w}+\frac{C}{1-e^{-w \alpha}}\right) \leq L_{0}$ and $\frac{C_{L_{0}}+M}{w}+\frac{C+M}{1-e^{-w \alpha}} \leq \frac{1}{2}$.

(A2) For each compact set $K \subseteq X, f(t, x) \in A P_{T}(R \times X, X)$ is uniformly continuous in $x \in K$ uniformly in $t \in R ; I_{i}(x)$ is almost periodic in $i \in Z$ uniformly in $x \in K$ and is a uniformly continuous function defined on the set $K \subseteq X$ for all $i \in Z$. 
(A3) Let $\left\{x_{n}\right\} \subseteq A P_{T}(R, X)$ be uniformly bounded in $R$ and uniformly convergent in each compact set of $R$, then $\left\{f\left(\cdot, x_{n}(\cdot)\right)\right\}$ is relatively compact in $P C(R, X)$.

(A4) There exists a compact set $K_{0} \subseteq X$, for any $r>0$,

$$
B_{r}=\left\{u \in A P_{T}(R, X):\|u\| \leq L_{0},\|u(t+\tau)-u(t)\| \leq r,\left|t-t_{i}\right|>r, i \in Z, \tau \in \Omega_{r, f, I_{i}, K_{0}, T}\right\},
$$

such that $R(x) \subseteq K_{0}$ for all $x \in B_{r}$, where the choice of $\Omega_{r, f, I_{i}, K_{0}, T}$ is similar to that of Lemma 2.5 .

Theorem 3.1 Suppose the conditions (H), (A1)-(A4) hold, then (1.3) has a mild piecewise almost periodic solution.

Proof Define the operator $\Gamma$ on $A P_{T}(R, X)$ by

$$
\Gamma u(t)=\int_{-\infty}^{t} T(t, s) f(s, u(s)) d s+\sum_{t_{i}<t} T\left(t, t_{i}\right) I_{i}\left(u\left(t_{i}\right)\right) .
$$

Now we use Schauder's fixed point theorem to prove that $\Gamma$ has a fixed point in $A P_{T}(R, X)$.

For any $n \in N$, let

$$
B_{\frac{1}{n}}=\left\{u \in A P_{T}(R, X):\|u\| \leq L_{0},\|u(t+\tau)-u(t)\| \leq \frac{1}{n},\left|t-t_{i}\right|>\frac{1}{n}, i \in Z, \tau \in \Omega_{\frac{1}{n} f, I_{i}, K_{0}, T}\right\},
$$

and

$$
B=\bigcap_{n \in N} B_{\frac{1}{n}}
$$

By (A4), for any $n \in N,\left\{u(t): u \in B_{\frac{1}{n}}, t \in R\right\} \subseteq K_{0}$. Note that $\{u(t): u \in B, t \in R\} \subseteq\left\{u(t): u \in B_{\frac{1}{n}}, t \in R\right\}$, so $\{u(t): u \in B, t \in R\} \subseteq K_{0}$. By (A2), $\{f($, $\left.x): x \in K_{0}\right\}$ and $\left\{I_{i}(x): x \in K_{0}\right\}$ are uniformly piecewise almost periodic families. Then by Lemma 2.5, there exists a relatively dense set $\Omega_{\frac{1}{n}, f, I_{i}, K_{0}, T}$ of $R$. As the proof of [[28], Theorem 2.6] for the case of $A P(R, X)$, one shows that for each $n \in N$, the set ${ }^{B} \frac{1}{n}$ is relatively compact and so is $B$. Obviously, $B$ is a nonempty closed convex set. For any $u \in B$,

$$
\begin{aligned}
\|\Gamma u(t)\| & =\left\|\int_{-\infty}^{t} T(t, s) f(s, u(s)) d s+\sum_{t_{i}<t} T\left(t, t_{i}\right) I_{i}\left(u\left(t_{i}\right)\right)\right\| \\
& \leq \int_{-\infty}^{t} M e^{-w(t-s)}\|f(s, u(s))\| d s+\sum_{t_{i}<t} M e^{-w\left(t-t_{i}\right)}\left\|I_{i}\left(u\left(t_{i}\right)\right)\right\| \\
& \leq \frac{M}{w} C_{L 0}+\sum_{t_{i}<t} M e^{-w\left(t-t_{i}\right)} C .
\end{aligned}
$$

In order to estimate the second term on the right-hand side of the above formula, we assume $t_{j} \leq t<t_{j+1}, j \in Z$, so

$$
t-t_{i}=\left(t-t_{j}\right)+\left(t_{j}-t_{i}\right) \geq(j-i) \alpha,
$$


and,

$$
\sum_{t_{i}<t} M e^{-w\left(t-t_{i}\right)} C \leq \sum_{-\infty<i \leq j} M e^{-w(j-i) \alpha} C=\sum_{0 \leq k=j-i<\infty} M e^{-w k \alpha} C=\frac{M}{1-e^{-w \alpha}} C .
$$

Thus

$$
\|\Gamma u(t)\| \leq \frac{M}{w} C_{L_{0}}+\frac{M}{1-e^{-w \alpha}} C \leq L_{0} .
$$

By (A2), for any $n \in N$, there exists a number $0<\delta \leq \frac{1}{n}$, if $\|x-y\| \leq \delta$ then $\|f(t, x)-f(t, y)\| \leq \frac{1}{n}$ and $\left\|I_{i}(x)-I_{i}(y)\right\| \leq \frac{1}{n}$ for all $i \in Z$.

For any $u \in B \subset B_{\frac{1}{n}}(\forall n \in N)$ and $\tau \in \Omega_{\frac{1}{n}, f, I_{i}, K_{0}, T}$,

$$
\|u(t+\tau)-u(t)\| \leq \frac{1}{n}(=\delta) \quad\left(\left|t-t_{i}\right|>\frac{1}{n}\right) .
$$

So, for $\left|t-t_{i}\right|>\frac{1}{n}$

$$
\begin{aligned}
& \|f(t+\tau, u(t+\tau))-f(t, u(t))\| \\
& \quad \leq\|f(t+\tau, u(t+\tau))-f(t, u(t+\tau))\|+\|f(t, u(t+\tau))-f(t, u(t))\| \\
& \quad \leq \frac{1}{n}+\frac{1}{n}=\frac{2}{n} .
\end{aligned}
$$

Similarly, for $q \in Z$ with $\left|t_{i+q}-t_{i}-\tau\right|<\frac{1}{n}$, we have

$$
\left\|I_{i+q}\left(u\left(t_{i+q}\right)\right)-I_{i}\left(u\left(t_{i}\right)\right)\right\| \leq \frac{2}{n} .
$$

Then,

$$
\begin{aligned}
\Gamma u(t+\tau)-\Gamma u(t)= & \int_{-\infty}^{t+\tau} T(t+\tau, s) f(s, u(s)) d s+\sum_{t_{i}<t+\tau} T\left(t+\tau, t_{i}\right) I_{i}\left(u\left(t_{i}\right)\right) \\
& -\int_{-\infty}^{t} T(t, s) f(s, u(s)) d s-\sum_{t_{i}<t} T\left(t, t_{i}\right) I_{i}\left(u\left(t_{i}\right)\right) \\
= & \int_{-\infty}^{t} T(t+\tau, s+\tau) f(s+\tau, u(s+\tau)) d s \\
& +\sum_{t_{i}<t} T\left(t+\tau, t_{i}+\tau\right) I_{i+q}\left(u\left(t_{i+q}\right)\right) \\
& -\int_{-\infty}^{t} T(t, s) f(s, u(s)) d s-\sum_{t_{i}<t} T\left(t, t_{i}\right) I_{i}\left(u\left(t_{i}\right)\right) \\
= & \int_{-\infty}^{t}[T(t+\tau, s+\tau) f(s+\tau, u(s+\tau))-T(t, s) f(s, u(s))] d s \\
& +\sum_{t_{i}<t}\left[T\left(t+\tau, t_{i}+\tau\right) I_{i+q}\left(u\left(t_{i+q}\right)\right)-T\left(t, t_{i}\right) I_{i}\left(u\left(t_{i}\right)\right)\right] .
\end{aligned}
$$


By Lemmas 2.4, 2.5, Equations (3.1) and (3.2), we have

$$
\begin{aligned}
& \left\|\int_{-\infty}^{t}[T(t+\tau, s+\tau) f(s+\tau, u(s+\tau))-T(t, s) f(s, u(s))] d s\right\| \\
& =\int_{-\infty}^{t} T(t+\tau, s+\tau)[f(s+\tau, u(s+\tau))-f(s, u(s))] d s \\
& \quad+\int_{-\infty}^{t}[T(t+\tau, s+\tau)-T(t, s)] f(s, u(s)) d s \| \\
& \leq \int_{-\infty}^{t}\|T(t+\tau, s+\tau)\|\|f(s+\tau, u(s+\tau))-f(s, u(s))\| d s \\
& \quad+\int_{-\infty}^{t}\|T(t+\tau, s+\tau)-T(t, s)\|\|f(s, u(s))\| d s \\
& \leq \int_{-\infty}^{t} \frac{2}{n} M e^{-w(t-s)} d s+\int_{-\infty}^{t} \frac{1}{n} e^{-\frac{w(t-s)}{2}} C_{L 0} d s \\
& =\frac{2}{n w} M+\frac{2}{n w} C_{L 0},
\end{aligned}
$$

and

$$
\begin{aligned}
\| \sum_{t_{i}<t} & {\left[T\left(t+\tau, t_{i}+\tau\right) I_{i+q}\left(u\left(t_{i+q}\right)\right)-T\left(t, t_{i}\right) I_{i}\left(u\left(t_{i}\right)\right)\right] \| } \\
=\| & \sum_{t_{i}<t} T\left(t+\tau, t_{i}+\tau\right)\left[I_{i+q}\left(u\left(t_{i+q}\right)\right)-I_{i}\left(u\left(t_{i}\right)\right)\right] \\
& +\sum_{t_{i}<t}\left[T\left(t+\tau, t_{i}+\tau\right)-T\left(t, t_{i}\right)\right] I_{i}\left(u\left(t_{i}\right)\right) \| \\
\leq & \sum_{t_{i}<t}\left\|T\left(t+\tau, t_{i}+\tau\right)\right\|\left\|I_{i+q}\left(u\left(t_{i+q}\right)\right)-I_{i}\left(u\left(t_{i}\right)\right)\right\| \\
& +\sum_{t_{i}<t}\left\|T\left(t+\tau, t_{i}+\tau\right)-T\left(t, t_{i}\right)\right\|\left\|I_{i}\left(u\left(t_{i}\right)\right)\right\| \\
\leq & \sum_{t_{i}<t} M e^{-w\left(t-t_{i}\right)} \frac{2}{n}+\sum_{t_{i}<t} \frac{1}{n} e^{-\frac{w\left(t-t_{i}\right)}{2}} C \\
\leq & \frac{2}{n\left(1-e^{-w \alpha}\right)} M+\frac{2}{n\left(1-e^{-w \alpha}\right)} C .
\end{aligned}
$$

Therefore, for any $n \in N$, there exists a relatively dense set $\Omega_{\frac{1}{n}, f, I_{i}, K_{0}, T}$ of $R$, for

$$
\begin{gathered}
\|\Gamma u(t+\tau)-\Gamma u(t)\| \leq \frac{2 M+2 C_{L_{0}}}{n w}+\frac{2 M+2 C}{n\left(1-e^{-w \alpha}\right)} \leq \frac{1}{n}, \\
\|\Gamma u(t+\tau)-\Gamma u(t)\| \leq \frac{2 M+2 C_{L_{0}}}{n w}+\frac{2 M+2 C}{n\left(1-e^{-w \alpha}\right)} \leq \frac{1}{n} .
\end{gathered}
$$

That is, $\Gamma u \in B_{\frac{1}{n}}$. Since $n$ is arbitrary, $\Gamma u \in \cap_{n \in N} B_{\frac{1}{n}}=B$, that is $\Gamma B \subseteq B$. 
We will show $\Gamma$ is compact. To this end, first we show $\Gamma$ is continuous. In fact, By (A2), for the above $\frac{1}{n}, \delta>0$ for each compact set $K \subseteq X, x_{1}, x_{2} \in K$ and $\left\|x_{1}-x_{2}\right\| \leq \delta$, we have

$$
\left\|f\left(t, x_{1}\right)-f\left(t, x_{2}\right)\right\| \leq \frac{1}{n} \quad\left\|I_{i}\left(x_{1}\right)-I_{i}\left(x_{2}\right)\right\| \leq \frac{1}{n},
$$

for all $t \in R, i \in N$. Let $x, y \in B,\|x-y\| \leq \delta$. Since $R(x) \subseteq K_{0}$ and $R(y) \subseteq K_{0}$,

$$
\|f(t, x(t))-f(t, y(t))\| \leq \frac{1}{n} \quad\left\|I_{i}(x(t))-I_{i}(y(t))\right\| \leq \frac{1}{n} .
$$

So

$$
\begin{aligned}
\|\Gamma x(t)-\Gamma y(t)\|= & \| \int_{-\infty}^{t} T(t, s) f(s, x(s)) d s+\sum_{t_{i}<t} T\left(t, t_{i}\right) I_{i}\left(x\left(t_{i}\right)\right) \\
& -\int_{-\infty}^{t} T(t, s) f(s, y(s)) d s-\sum_{t_{i}<t} I_{i}\left(\gamma\left(t_{i}\right)\right) \| \\
\leq & \int_{-\infty}^{t}\|T(t, s)\|\|f(s, x(s))-f(s, y(s))\| d s \\
& +\sum_{t_{i}<t}^{t}\left\|T\left(t, t_{i}\right)\right\|\left\|I_{i}\left(x\left(t_{i}\right)\right)-I_{i}\left(\gamma\left(t_{i}\right)\right)\right\| \\
\leq & \int_{-\infty}^{M e^{-w(t-s)}}\|f(s, x(s))-f(s, y(s))\| d s \\
& +\sum_{t_{i}<t} M e^{-w\left(t-t_{i}\right)}\left\|I_{i}\left(x\left(t_{i}\right)\right)-I_{i}\left(\gamma\left(t_{i}\right)\right)\right\| \\
\leq & \frac{M}{w n}+\frac{M}{n\left(1-e^{-w \alpha}\right)} \rightarrow 0 \text { if } n \rightarrow \infty .
\end{aligned}
$$

Next, we show the following three statements:

(S1) $\{\Gamma u(t): u \in B\}$ is a relatively compact subset of $X$ for each $t \in R$.

(S2) $\{\Gamma u: u \in B\}$ is equicontinuous.

(S3) $\{\Gamma u: u \in B\}$ is equi-almost-periodic.

(S3) is obvious because $B$ is.

For any $\varepsilon>0$, let

$$
\begin{aligned}
\Gamma_{\varepsilon} u(t) & =\int_{-\infty}^{t-\varepsilon} T(t, s) f(s, u(s)) d s+\sum_{t_{i}<t-\varepsilon} T\left(t, t_{i}\right) I_{i}\left(u\left(t_{i}\right)\right) \\
& =T(t, t-\varepsilon)\left[\int_{-\infty}^{t-\varepsilon} T(t-\varepsilon, s) f(s, u(s)) d s+\sum_{t_{i}<t-\varepsilon} T\left(t-\varepsilon, t_{i}\right) I_{i}\left(u\left(t_{i}\right)\right)\right] \\
& =T(t, t-\varepsilon) \Gamma u(t-\varepsilon) .
\end{aligned}
$$

Since $\{\Gamma u(t-\varepsilon): u \in B\}$ is uniformly bounded in $X$ and $T(t, t-\varepsilon)$ is compact, so $\left\{\Gamma_{\varepsilon} u\right.$ $(t): u \in B\}$ is relatively compact in $X$. Moreover, 


$$
\begin{aligned}
\left\|\Gamma u(t)-\Gamma_{\varepsilon} u(t)\right\| & =\left\|\int_{t-\varepsilon}^{t} T(t, s) f(s, x(s)) d s+\sum_{t-\varepsilon<t_{i}<t} T\left(t, t_{i}\right) I_{i}\left(u\left(t_{i}\right)\right)\right\| \\
& \leq \int_{t-\varepsilon}^{t}\|T(t, s)\|\|f(s, x(s))\| d s+\sum_{t-\varepsilon<t_{i}<t}\left\|T\left(t, t_{i}\right)\right\|\left\|I_{i}\left(u\left(t_{i}\right)\right)\right\| \\
& \leq \int_{t-\varepsilon}^{t} M e^{-w(t-s)}\|f(s, x(s))\| d s+\sum_{t-\varepsilon<t_{i}<t} M e^{-w\left(t-t_{i}\right)}\left\|I_{i}\left(u\left(t_{i}\right)\right)\right\| \\
& \leq \varepsilon \frac{M}{w} C_{L_{0}}+\varepsilon \frac{M}{\alpha} C .
\end{aligned}
$$

So, $\{\Gamma u(t): u \in B\}$ is a relatively compact subset of $X$ for each $t \in R$. This show (S1). It remains to prove (S2). Let $t^{\prime \prime}<t^{\prime}, t^{\prime \prime}, t^{\prime} \in R, u \in B$,

$$
\begin{aligned}
\Gamma u\left(t^{\prime}\right)-\Gamma u\left(t^{\prime \prime}\right)= & \int_{-\infty}^{t^{\prime}} T\left(t^{\prime}, s\right) f(s, u(s)) d s+\sum_{t_{i}<t^{\prime}} T\left(t^{\prime}, t_{i}\right) I_{i}\left(u\left(t_{i}\right)\right) \\
& -\int_{-\infty}^{t^{\prime \prime}} T\left(t^{\prime \prime}, s\right) f(s, u(s)) d s-\sum_{t_{i}<t^{\prime \prime}} T\left(t^{\prime \prime}, t_{i}\right) I_{i}\left(u\left(t_{i}\right)\right) \\
= & \int_{-\infty}^{t^{\prime \prime}}\left[T\left(t^{\prime}, s\right)-T\left(t^{\prime \prime}, s\right)\right] f(s, u(s)) d s \\
& +\int_{t^{\prime \prime}}^{t^{\prime}} T\left(t^{\prime}, s\right) f(s, u(s)) d s \\
& +\sum_{t_{i}<t^{\prime \prime}}\left[T\left(t^{\prime}, t_{i}\right)-T\left(t^{\prime \prime}, t_{i}\right)\right] I_{i}\left(u\left(t_{i}\right)\right) \\
& +\sum_{t^{\prime \prime}<t_{i}<t^{\prime}} T\left(t^{\prime}, t_{i}\right) I_{i}\left(u\left(t_{i}\right)\right) .
\end{aligned}
$$

Moreover,

$$
\begin{aligned}
& \int_{-\infty}^{t^{\prime \prime}}\left[T\left(t^{\prime}, s\right)-T\left(t^{\prime \prime}, s\right)\right] f(s, u(s)) d s \\
= & \int_{0}^{\infty}\left[T\left(t^{\prime}, t^{\prime \prime}-s\right)-T\left(t^{\prime \prime}, t^{\prime \prime}-s\right)\right] f\left(t^{\prime \prime}-s, u\left(t^{\prime \prime}-s\right)\right) d s \\
= & \int_{0}^{\infty}\left[T\left(t^{\prime}, t^{\prime \prime}\right) T\left(t^{\prime \prime}, t^{\prime \prime}-s\right)-T\left(t^{\prime \prime}, t^{\prime \prime}-s\right)\right] f\left(t^{\prime \prime}-s, u\left(t^{\prime \prime}-s\right)\right) d s \\
= & \int_{0}^{\infty}\left[T\left(t^{\prime}, t^{\prime \prime}\right)-I\right] T\left(t^{\prime \prime}, t^{\prime \prime}-s\right) f\left(t^{\prime \prime}-s, u\left(t^{\prime \prime}-s\right)\right) d s .
\end{aligned}
$$

By (H6), for the given $\varepsilon>0$, there exists $\delta(\varepsilon)<\frac{\varepsilon}{4 M\left(C_{L_{0}}+\frac{C}{\alpha}\right)}$ such that if $t^{\prime}, t^{\prime \prime}$ belongs to a same continuity and $0<t^{\prime}-t^{\prime \prime}<\delta$, then 


$$
\left\|T\left(t^{\prime}, t^{\prime \prime}\right)-I\right\|<\min \left\{\frac{w \varepsilon}{4 M C_{L_{0}}}, \frac{\left(1-e^{-w \alpha}\right) \varepsilon}{4 M C}\right\} .
$$

So,

$$
\begin{aligned}
& \left\|\int_{-\infty}^{t^{\prime \prime}}\left[T\left(t^{\prime}, s\right)-T\left(t^{\prime \prime}, s\right)\right] f(s, u(s)) d s\right\| \\
& \quad \leq \int_{0}^{\infty}\left\|T\left(t^{\prime}, t^{\prime \prime}\right)-I\right\|\left\|T\left(t^{\prime \prime}, t^{\prime \prime}-s\right)\right\|\left\|f\left(t^{\prime \prime}-s, u\left(t^{\prime \prime}-s\right)\right)\right\| d s \\
& \quad \leq \int_{0}^{\infty} \frac{w \varepsilon}{4 M C_{L_{0}}} M e^{-w s} C_{L_{0}} d s<\frac{\varepsilon}{4},
\end{aligned}
$$

and,

$$
\left\|\int_{t^{\prime \prime}}^{t^{\prime}} T\left(t^{\prime}, s\right) f(s, u(s)) d s\right\| \leq \int_{t^{\prime \prime}}^{t^{\prime}}\left\|T\left(t^{\prime}, s\right)\right\|\|f(s, u(s)) d s\|<\delta M C_{L_{0}}<\frac{\varepsilon}{4} .
$$

Similarly,

$$
\begin{aligned}
& \left\|\sum_{t_{i}<t^{\prime \prime}}\left[T\left(t^{\prime}, t_{i}\right)-T\left(t^{\prime \prime}, t_{i}\right)\right] I_{i}\left(u\left(t_{i}\right)\right)\right\| \\
& =\left\|\sum_{t_{i}<t^{\prime \prime}}\left[T\left(t^{\prime}, t^{\prime \prime}\right) T\left(t^{\prime \prime}, t_{i}\right)-T\left(t^{\prime \prime}, t_{i}\right)\right] I_{i}\left(u\left(t_{i}\right)\right)\right\| \\
& =\left\|\sum_{t_{i}<t^{\prime \prime}}\left[T\left(t^{\prime}, t^{\prime \prime}\right)-I\right] T\left(t^{\prime \prime}, t_{i}\right) I_{i}\left(u\left(t_{i}\right)\right)\right\| \\
& \leq \sum_{t_{i}<t^{\prime \prime}}\left\|T\left(t^{\prime}, t^{\prime \prime}\right)-I\right\|\left\|T\left(t^{\prime \prime}, t_{i}\right)\right\|\left\|I_{i}\left(u\left(t_{i}\right)\right)\right\| \\
& \leq \sum_{t_{i}<t^{\prime \prime}} \frac{\left(1-e^{-w \alpha}\right) \varepsilon}{4 M C} M e^{-w\left(t^{\prime \prime}-t_{i}\right)} C \leq \frac{\varepsilon}{4},
\end{aligned}
$$

and,

$$
\left\|\sum_{t^{\prime \prime}<t_{i}<t^{\prime}} T\left(t^{\prime}, t_{i}\right) I_{i}\left(u\left(t_{i}\right)\right)\right\| \leq \sum_{t^{\prime \prime}<t_{i}<t^{\prime}}\left\|T\left(t^{\prime}, t_{i}\right)\right\|\left\|I_{i}\left(u\left(t_{i}\right)\right)\right\|<\frac{\delta}{\alpha} M C<\frac{\varepsilon}{4} .
$$

Thus, for $u \in B$, when $t^{\prime}, t^{\prime \prime} \in R$ belongs to a same continuity and $0<t^{\prime}-t^{\prime \prime}<\delta$,

$$
\left\|\Gamma u\left(t^{\prime}\right)-\Gamma u\left(t^{\prime \prime}\right)\right\| \leq \varepsilon .
$$

That is, $\{\Gamma u: u \in B\}$ is equicontinuous and (S2) holds.

Now the conditions of Lemma 2.7 are met, so $\Gamma$ is compact. By Schauder's point fixed theorem, $\Gamma$ has a fixed point $u \in B$. That is, (1.3) has a mild piecewise almost periodic solution $u(t)$.

The proof is complete. 
Remark 3.2 As we pointed out in Introduction, to get the almost periodic solutions of impulsive differential equations, most people use the Contraction theorem. To be different, we use Schauder's fixed point theorem. This makes proofs difficult. In this case, we impose a stronger condition (A4). To investigate this problem without (A4) and give a easy example will be our next study.

Remark 3.3 Note that the uniform continuity is weaker than the Lipschitz continuity So if the conditions of uniformly continuity of $f$ and $I_{i}$ in (A2) is replaced by

$$
\|f(t, x)-f(t, y)\|+\left\|I_{i}(x)-I_{i}(y)\right\| \leq \eta\|x-y\|
$$

for all $x, y \in X, \eta \in(0,1)$, we can also get the mild piecewise almost periodic solution to (1.3) by Theorem 3.1.

In the end, we will use generalized Gronwall-Bellman inequality (Lemma 2.9) to show the asymptotic stability of piecewise almost periodic solutions to (1.3) under the Lipschitz condition.

Theorem 3.4 Assume the conditions of Theorem 3.1 are fulfilled except that (A2) is replaced by (3.3). Assume further that $M \eta-w<0$, then (1.3) has an asymptotically stable almost periodic solution.

Proof By Remark 3.3, (1.3) has a mild piecewise almost periodic solution $x(t)$,

$$
x(t)=T(t, \sigma) x(\sigma)+\int_{\sigma}^{t} T(t, s) f(s, x(s)) d s+\sum_{\sigma<t_{i}<t} T\left(t, t_{i}\right) I_{i}\left(u\left(t_{i}\right)\right) .
$$

Let $u(t)=u(t, \sigma, \phi)$ and $v(t)=v(t, \sigma, \psi)$ be two solutions of Equation (1.3), then

$$
\begin{aligned}
& u(t)=T(t, \sigma) \varphi+\int_{\sigma}^{t} T(t, s) f(s, u(s)) d s+\sum_{\sigma<t_{i}<t} T\left(t, t_{i}\right) I_{i}\left(u\left(t_{i}\right)\right), \\
& v(t)=T(t, \sigma) \psi+\int_{\sigma}^{t} T(t, s) f(s, v(s)) d s+\sum_{\sigma<t_{i}<t} T\left(t, t_{i}\right) I_{i}\left(v\left(t_{i}\right)\right) .
\end{aligned}
$$

So

$$
\begin{aligned}
\|u(t)-v(t)\|= & \| T(t, \sigma) \varphi-T(t, \sigma) \psi+\int_{\sigma}^{t} T(t, s)[f(s, u(s))-f(s, v(s))] d s \\
& +\sum_{\sigma<t_{i}<t} T\left(t, t_{i}\right)\left[I_{i}\left(u\left(t_{i}\right)\right)-I_{i}\left(v\left(t_{i}\right)\right)\right] \| \\
\leq & \|T(t, \sigma)\|\|\varphi-\psi\|+\int_{\sigma}^{t}\|T(t, s)\|\|f(s, u(s))-f(s, v(s))\| d s \\
& +\sum_{\sigma<t_{i}<t}\left\|T\left(t, t_{i}\right)\right\|\left\|I_{i}\left(u\left(t_{i}\right)\right)-I_{i}\left(v\left(t_{i}\right)\right)\right\| \\
\leq & M e^{-w(t-\sigma)}\|\varphi-\psi\|+\int_{\sigma}^{t} M \eta e^{-w(t-s)}\|u(s)-v(s)\| d s \\
& +\sum_{\sigma<t_{i}<t} M \eta e^{-w\left(t-t_{i}\right)}\left\|u\left(t_{i}\right)-v\left(t_{i}\right)\right\| .
\end{aligned}
$$


Then,

$$
\begin{aligned}
e^{w t}\|u(t)-v(t)\| & \leq M e^{w \sigma}\|\varphi-\psi\|+\int_{\sigma}^{t} M \eta e^{w s}\|u(s)-v(s)\| d s \\
& +\sum_{\sigma<t_{i}<t} M \eta e^{w t_{i}}\left\|u\left(t_{i}\right)-v\left(t_{i}\right)\right\| .
\end{aligned}
$$

Let $\Upsilon(t)=e^{w t}|| u(t)-v(t) \|$, then

$$
\Upsilon(t) \leq M \Upsilon(\sigma)+\int_{\sigma}^{t} M \eta \Upsilon(s) d s+\sum_{\sigma<t_{i}<t} M \eta \Upsilon\left(t_{i}\right) .
$$

By Lemma 2.9, we have

$$
\Upsilon(t) \leq M \Upsilon(\sigma) \prod_{\sigma<t_{i}<t}(1+M \eta) e^{\int_{\sigma}^{t} M \eta d s}=M \Upsilon(\sigma) \prod_{\sigma<t_{i}<t}(1+M \eta) e^{M \eta(t-\sigma)},
$$

that is,

$$
\|u(t)-v(t)\| \leq M\|\varphi-\psi\| \coprod_{\sigma<t_{i}<t}(1+M \eta) e^{(M \eta-w)(t-\sigma)} .
$$

This completes the proof.

\section{Conclusion}

The aim of this article was to give sufficient conditions for existence and asymptotic stability of almost periodic solutions for abstract impulsive differential equations. By taking into consideration the relative compactness for subsets in $A P_{T}(R, X)$, we get almost periodic solutions to impulsive differential equation. In addition, we use generalized Gronwall-Bellman inequality to show the above solution is asymptotically stable.

\section{Acknowledgements}

This research was supported by the National Natural Science Foundation of China (No. 11071048). The authors would like to thank the referees for carefully reading the manuscript, giving valuable comments and suggestions on the topic of this article.

\section{Authors' contributions}

$J$ carried out the main part of this manuscript. CZ participated discussion and corrected the main theorem. All authors read and approved the final manuscript.

\section{Competing interests}

The authors declare that they have no competing interests.

Received: 12 November 2011 Accepted: 20 March 2012 Published: 20 March 2012

\section{References}

1. Samoilenko, AM, Perestyuk, NA: Impulsive differential equations. World Scientific, Singapore (1995)

2. Cuevas, C, Hernandez, E, Rabelo, M: The existence of solutions for impulsive neutral functional differential equations. Comput Math Appl. 58(4):744-757 (2009)

3. Cuevas, C, N'Guerekata, GM, Rabelo, M: Mild solutions for impulsive neutral functional differential equations with statedependent delay. Semigroup Forum. 80, 375-390 (2010)

4. dos Santos, JPC, Arjunan, M, Cuevas, C: Existence results for fractional neutral integro-differential equations with statedependent delay. Comput Math Appl. 62, 1275-1283 (2011)

5. dos Santos, JPC, Cuevas, C, de Andrade, B: Existence results for a fractional equation with state-dependent delay. Adv Diff Equ (2007). doi:10.1155/2011/642013

6. Stamov, GT, Alzabut, JO, Atanasov, P, Stamov, AG: Almost periodic solutions for an impulsive delay model of price fluctuations in commodity markets. Nonlinear Anal RWA. 12, 3170-3176 (2011)

7. Wang, J, Xiang, X, Wei, W: Linear impulsive periodic system with time-varying generating operators on Banach space. Adv Differ Equ (2007). doi:10.1155/2007/26196 
8. Alzabut, JO, Stamov, GT, Sermutlu, E: On almost periodic solutions for an impulsive delay logarithmic population model. Math Comput Model. 51, 625-631 (2010)

9. Hernandez, EM, Tanaka Aki, SM, Henriquez, H: Global solutions for impulsive abstract partial differential equations. Comput Math Appl. 56, 1206-1215 (2008)

10. Akhmetov, MU, Zafer, A: Stability of the zero solution of impulsive differential equations by the Lyapunov second method. J Math Anal Appl. 248, 69-82 (2000)

11. Ignatyev, AO: On the stability of invariant sets of systems with impulse effect. Nonlinear Anal TMA. 69, 53-72 (2008)

12. Wang, J, Xiang, X, Peng, Y: Periodic solutions of semilinear impulsive periodic system on Banach space. Nonlinear Anal TMA. 71, e1344-e1353 (2009)

13. Liang, J, Liu, J, Xiao, T: Periodic solutions of delay impulsive differential equations. Nonlinear Anal TMA. 74, 6835-6842 (2011)

14. Stamov, GT: Asymptotic stability of almost periodic systems of impulsive differential-difference equations. Asymptotic Anal. 27, 1-8 (2001)

15. Yang, Z, Xu, D: Existence and exponential stability of periodic solution for impulsive delay differential equations and applications. Nonlinear Anal TMA. 64, 130-145 (2006)

16. Sivasankaran, $\mathrm{S}$, Arjunan, M, Vijayakumar, V: Existence of global solutions for second order impulsive abstract partial differential equations. Nonlinear Anal TMA. 74, 6747-6757 (2011)

17. Stamov, GT, Alzabut, JO: Almost periodic solutions for abstract impulsive differential equations. Nonlinear Anal TMA. 72 2457-2464 (2010)

18. Henriquez, HR, de Andrade, B, Rabelo, M: Existence of almost periodic solutions for a class of abstract impulsive differential equations. ISRN Math (2011). doi:10.5402/2011/632687

19. Chang, Y, Zhao, Z, N'Guerekata, GM: A new composition theorem for square-mean almost automorphic functions and applications to stochastic differential equations. Nonlinear Anal TMA. 74, 2210-2219 (2011)

20. Li, H, Huang, F, Li, J: Composition of pseudo almost-periodic functions and semilinear differential equations. J Math Anal Appl. 255, 436-446 (2001)

21. Agarwal, RP, de Andrade, B, Cuevas, C: Weighted pseudo-almost periodic solutions of a class of semilinear fractional differential equations. Nonlinear Anal RWA. 11, 3532-3554 (2010)

22. Agarwal, RP, Cuevas, C, dos Santos, JPC: Analytic resolvent operator and existence results for fractional integrodifferential equations. J Abst Diff Equa Appl. 2(2):26-47 (2012)

23. Agarwal, RP, de Andrade, B, Cuevas, C, Henriquez, E: Asymptotic periodicity for some classes of integro-differential equations and applications. Adv Math Sci Appl. 21(1):1-31 (2011)

24. Pazy, A: Semigroups of Linear Operators and Applications to Partial Differential Equations. Springer, New York (1983)

25. Schnaubelt, R, lannelli, M, Nagel, R, Piazzera, S: Asymptotic Behaviour of Parabolic Nonautonomous Evolution Equation. In Lecture Notes in Math, vol. 1855, pp. 401-472.Springer-Verlag, Berlin (2004). Functional Analytic Methods for Evolution Equations

26. Corduneanu, C: Almost Periodic Oscillations and Waves. Springer, New York (2009)

27. Wiggins, S: Introduction to Applied Nonlinear Dynamical Systems and Chaos. Springer, New York (1990)

28. Fink, AM: Almost Periodic Differential Equations. In Lecture Notes in Mathematics, vol. 377,Springer-Verlag, New York (1974)

doi:10.1186/1687-1847-2012-34

Cite this article as: Liu and Zhang: Existence and stability of almost periodic solutions for impulsive differential equations. Advances in Difference Equations 2012 2012:34.

\section{Submit your manuscript to a SpringerOpen ${ }^{\circ}$ journal and benefit from:}

- Convenient online submission

- Rigorous peer review

- Immediate publication on acceptance

- Open access: articles freely available online

- High visibility within the field

- Retaining the copyright to your article

Submit your next manuscript at $\boldsymbol{s p r i n g e r o p e n . c o m ~}$ 